\title{
EBERLEIN-ŠMULYAN THEOREM FOR ABELIAN TOPOLOGICAL GROUPS
}

\author{
MONTSERRAT BRUGUERA, ELENA MARTÍN-PEINADOR \\ AND VAJA TARIELADZE
}

\begin{abstract}
Leaning on a remarkable paper of Pryce, the paper studies two independent classes of topological Abelian groups which are strictly angelic when endowed with their Bohr topology. Some extensions are given of the Eberlein-Šmulyan theorem for the class of topological Abelian groups, and finally, for a large subclass of the latter, Bohr angelicity is related to the Schur property.
\end{abstract}

\section{Introduction}

In many branches of mathematics the notion of compactness appears as a fundamental tool, and the optimal situation is when it can be used in its sequential version. Unfortunately this is not always the case, and there is a strong need to look for classes of topological spaces where compactness is equivalent to sequential or countable compactness. It was known from the early developments of general topology that metrizable spaces have this property. An important class of nonmetrizable spaces for which the equivalence also holds is provided by infinitedimensional Banach spaces endowed with their weak topology. In fact, the EberleinŠmulyan theorem essentially states that a subset $K$ of a Banach space is weakly compact if and only if it is sequentially weakly compact. Grothendieck was able to extend a part of this statement to the larger class of locally convex spaces which are quasi-complete in their Mackey topology. He proved that within the mentioned class, relatively countably weak compactness is equivalent to relatively weak compactness.

Let us observe that for an Abelian topological group $G$, there is a topology which can be considered analogous to the weak topology in the context of topological vector spaces, namely, the topology induced by the set $\Gamma G$ of all continuous characters of $G$. We will denote it by $\sigma(G, \Gamma G)$. In the literature it is frequently called the Bohr topology, since it coincides with the topology induced in $G$ from the Bohr compactification of $G$, a notion which goes back to the Danish mathematician Bohr. The Bohr compactification exists for any topological group, Abelian or not. The above given direct definition of the Bohr topology for Abelian groups is the one we will use henceforth.

The central core of this paper is the extension of the theorems of Eberlein, Šmulyan and Grothendieck to the class of Abelian dually separated groups

2000 Mathematics Subject Classification 22A05, 43A40, 54H11 (primary), 46A11, 46A50 (secondary).

The three authors were partially supported by DGICYT BFM 2003-05878. 
(Sections 3 and 4). Some first results in this direction were obtained in [6]. Our approach leans on the fact that for certain pairs of topological spaces $X, Y$, the space of continuous functions $C(X, Y)$ under the pointwise convergence topology is angelic. We will apply this kind of general 'angelicity' result to topological Abelian groups.

\section{Angelic spaces: definition and properties}

The term 'angelic space' was introduced by Fremlin to denominate, roughly speaking, those topological spaces for which various types of compactness are equivalent. Before the formal definition of angelic space, we introduce the notions it deals with and some results that are convenient for our subsequent developments. In what follows, $\mathbb{N}, \mathbb{R}$ and $\mathbb{C}$ stand for the sets of natural, real and complex numbers with their ordinary topological and algebraic structures. Also $\aleph_{0}:=\operatorname{card}(\mathbb{N})$ and $\mathfrak{c}:=\operatorname{card}(\mathbb{R})$.

A subset $A$ of a topological space $X$ is

(1) relatively compact if its closure $\bar{A}$ is compact;

(2) relatively countably compact if each sequence in $A$ has a cluster point in $X$;

(3) relatively sequentially compact if each sequence in $A$ has a subsequence converging to a point of $X$;

(4) countably compact, sequentially compact if in the above two definitions the cluster or limit point is required to be in $A$;

(5) sigma-compact if it is the union of countably many compact subsets.

Clearly, every compact or sequentially compact subset is countably compact. A similar assertion holds for the corresponding relative properties, and it is well known that the reverse implications hold in a metrizable space. In general, a sequentially compact subset of a completely regular Hausdorff space may be nonclosed, and its closure may not be countably compact (see Example 29).

A (completely regular) Hausdorff topological space is called

(1) a $g$-space if its relatively countably compact subsets are relatively compact [1] (in [18, Chapter 5, Part 3, §2, Exercise 1, p. 209], the term '(E)-space' is used for the same notion);

(2) a $g_{w}$-space if its countably compact subsets are relatively compact;

(3) an IN-space if its countably compact subsets are closed (in [21], the term 'C-space' is used for the same notion);

(4) an $\breve{S}$-space if its compact subsets are sequentially compact.

Clearly, if $X$ is a $g$-space, then it is a $g_{w}$-space. Observe also that for a Hausdorff $g_{w}+\mathrm{IN}$-space $X$, the countable compact subsets of $X$ are compact. A compact Hausdorff space is trivially a $g$-space. However, it may be neither an IN-space nor an $\check{\text { S-space. }}$

A Hausdorff topological space $X$ is said to be an angelic space if for every relatively countably compact subset $A$ of $X$ the following two claims hold.

(i) $A$ is relatively compact.

(ii) If $b \in \bar{A}$, then there is a sequence in $A$ that converges to $b$.

It can be said that a Hausdorff topological space $X$ is angelic if and only if $X$ is a $g$-space for which any compact subspace is a Frechet-Urysohn space. As proved in $[\mathbf{1}]$, an angelic space is exactly a hereditary $g$-space. 
Proposition 1 [26, Lemma 0.3]. If $X$ is an angelic space, and $A \subset X$, the following assertions are equivalent.

(1) $A$ is countably compact.

(2) $A$ is sequentially compact.

(3) $A$ is compact.

Using this statement it is straightforward to check the following proposition.

Proposition 2. If $X$ is an angelic space, then it is a $g+I N+\check{S}$-space.

We will see below that angelic spaces have quite good stability properties; nevertheless, the product of two angelic spaces may not be angelic. This fact motivated Govaerts [16] to define the strictly angelic spaces as those angelic spaces in which every separable compact subset is first countable. Notice that this new class is closed under countable products (Lemma 4).

Denote by $C(X, Y)$ the set of all continuous functions between the topological spaces $X$ and $Y$, and by $C_{\mathrm{p}}(X, Y)$ the same set endowed with the pointwise convergence topology.

The properties of angelic and of strictly angelic spaces listed jointly in the next lemma are proved in [26] and [16], respectively.

Lemma 3. (a) A homeomorph of a (strictly) angelic space is (strictly) angelic.

(b) Any subspace of a (strictly) angelic space is (strictly) angelic.

(c) If there is a continuous one-to-one map $f$ from a regular space $E$ into a (strictly) angelic space $X$, then $E$ is also (strictly) angelic.

(d) Let $\tau_{1}$ and $\tau_{2}$ be regular topologies in $X$ such that $\tau_{1} \leqslant \tau_{2}$. If $\left(X, \tau_{1}\right)$ is (strictly) angelic, $\left(X, \tau_{2}\right)$ is also (strictly) angelic.

(e) If $X$ is a completely regular Hausdorff space such that $C_{\mathrm{p}}(X, \mathbb{R})$ is (strictly) angelic and $Y$ is a metrizable space, then $C_{\mathrm{p}}(X, Y)$ is also (strictly) angelic.

On the other hand, we have the following lemma.

LEMma 4. The product of countably many strictly angelic spaces is strictly angelic.

A proof of Lemma 4 is provided in [16]; [5] provides (under the continuum hypothesis) an example of a nonangelic product of two compact Hausdorff angelic spaces.

We now establish a result that is important for our further work. It is based upon the significant result of Pryce mentioned in the abstract.

Proposition $5[\mathbf{1 6}, \mathbf{2 6}]$. Let $X$ be a Hausdorff topological space and let $Y$ be a metrizable topological space. If

$$
X=\overline{\bigcup_{n=1}^{\infty} K_{n},}
$$

where each $K_{n}(n=1,2, \ldots)$ is a relatively countably compact subset of $X$, then $C_{\mathrm{p}}(X, Y)$ is a strictly angelic space. 
Proof. Following [16], let us call a space $X$ sa-producing if $C_{\mathrm{p}}(X, \mathbb{R})$ is strictly angelic.

Put $X_{n}:=\overline{K_{n}}, n=1,2, \ldots$ Clearly, $K_{n}$ is dense and relatively countably compact in $X_{n}$. By [16, Theorem 2], $X_{n}$ is sa-producing. Consider $D:=\bigcup_{n=1}^{\infty} X_{n}$ equipped with the topology induced from $X$. Then, by [16, Theorem 3(b)], $D$ is sa-producing. The set $D$ is dense in $X$ as shown by the inclusion $\bigcup_{n=1}^{\infty} K_{n} \subset$ $\bigcup_{n=1}^{\infty} X_{n}=D$. Since $X$ contains a dense sa-producing subspace, $X$ itself is sa-producing according to $[\mathbf{1 6}$, Theorem $3(\mathrm{a})]$. Finally, take into account Lemma $3(\mathrm{e})$.

The fact that $C_{\mathrm{p}}(X, \mathbb{R})$ is a $g$-space for any countably compact $X$ had already been established in $[\mathbf{1 7}$, Theorem 1].

We shall say that a completely regular Hausdorff space $X$ is angelic-producing (or a Grothendieck space $[\mathbf{1}, \S 2])$ if $C_{\mathrm{p}}(X, \mathbb{R})$ is angelic. Whether any angelic-producing space is sa-producing too seems to be unknown.

Further study of angelic-producing spaces has been carried out in $[\mathbf{1}, \mathbf{8}, \mathbf{1 9}, \mathbf{2 5}]$ and some other papers. A general description of those spaces is posed as a problem in $[\mathbf{1}, 2.22]$.

\section{The Bohr topology for a topological group}

Let $G$ be a topological Abelian group, and let $\mathbb{T}$ be the unit circle of the complex plane with its multiplicative structure and the topology induced by the usual of $\mathbb{C}$. The group of all continuous homomorphisms from $G$ into $\mathbb{T}$ with pointwise operation will be denoted by $\Gamma G$. Any member of $\Gamma G$ is called a continuous character (of $G$ ). If $\Gamma G$ separates the points of $G$, then we will say that $G$ is dually separated or $G$ is a $D S$-group or $G$ has sufficiently many continuous characters.

The symbol $G^{\wedge}$ will denote $\Gamma G$ endowed with the compact-open topology, that is, the dual group of $G$. We put $G^{\wedge \wedge}:=\left(G^{\wedge}\right)^{\wedge}$, and this topological Abelian group is the bidual of $G$.

For a fixed $g \in G$, let $g^{\natural}: \Gamma G \longmapsto \mathbb{T}$ be the evaluation mapping $\varphi \longrightarrow \varphi(g)$ and $G^{\natural}:=\left\{g^{\natural}: g \in G\right\}$. It is easy to see that $G^{\natural} \subset \Gamma G^{\wedge}$ and therefore the equality $\alpha_{G}(g)=g^{\natural}$ defines a natural mapping from $G$ into $\Gamma G^{\wedge}$ which is a group homomorphism. If $\alpha_{G}$ is a topological isomorphism between $G$ and the bidual $G^{\wedge \wedge}$, the group $G$ is called reflexive.

Let $A \subset G, B \subset \Gamma G$ be nonempty subsets. The annihilator of $A$ is defined as

$$
A^{\perp}:=\{\phi \in \Gamma G: \phi(x)=1, \forall x \in A\} .
$$

The polars of $A$ and $B$ are

$$
A^{\triangleright}:=\{\phi \in \Gamma G: \operatorname{Re}(\phi(x)) \geqslant 0, \forall x \in A\}
$$

and

$$
B^{\triangleleft}:=\{x \in G: \operatorname{Re}(\phi(x)) \geqslant 0, \forall \phi \in B\},
$$

respectively, where Re stands for the real part.

It is easy to prove that the collection $\left\{K^{\triangleright}: K \subset G, K\right.$ nonempty compact $\}$ is a fundamental system of neighborhoods of the neutral element in $G^{\wedge}$.

A subset $A$ of $G$ is called quasi-convex if, for every $x \in G \backslash A$, there is some $\chi \in A^{\triangleright}$ such that $\operatorname{Re}(\chi(x))<0$. The quasi-convex hull of any subset $H \subset G$ is defined as 
the intersection of all quasi-convex subsets of $G$ containing $H$. It coincides with the set $Q(H):=\left(H^{\triangleright}\right)^{\triangleleft}$.

The group $G$ is locally quasi-convex if it has a neighborhood basis of the neutral element 0 , given by quasi-convex sets. Any Hausdorff locally quasi-convex group is dually separated. Observe also that the dual $G^{\wedge}$ of $G$ is locally quasi-convex. This implies that any reflexive group is locally quasi-convex.

The Bohr topology in $G$ is the coarsest topology of all those that make continuous the elements of $\Gamma G$. It is a group topology and it will be denoted by $\sigma(G, \Gamma G)$. The notation $G^{+}:=(G, \sigma(G, \Gamma G))$ is taken from $[\mathbf{1 0}]$ (where several delicate properties of the topological structure of $G^{+}$are also obtained). It is known that $G^{+}$is a totally bounded topological group. The case when $G:=E$ is a real topological vector space deserves special attention, for we then also have the weak topology $\sigma(E, L E)$, which is the topology generated in $E$ by the set $L E$ of all continuous linear functionals. As an Abelian group, $E$ may be endowed with the Bohr topology $\sigma(E, \Gamma E)$, and from the equality $\Gamma E=\{\exp (i f): f \in L E\}$, it follows that $\sigma(E, \Gamma E) \subset \sigma(E, L E)$. This fact implies that, whenever $E^{+}$is angelic, $(E, \sigma(E, L E))$ is also angelic, provided that $E$ is dually separated. Thus our results in the context of topological groups imply (and in some cases refine too) the analogous statements known to hold for locally convex spaces equipped with the weak topology.

The symbol $\sigma(\Gamma G, G)$ will denote the topology on $\Gamma G$ of pointwise convergence on the elements of $G$, while $\Gamma_{\sigma} G:=(\Gamma G, \sigma(\Gamma G, G))$. Observe that $\Gamma_{\sigma} G$ is totally bounded as a topological subgroup (in general nonclosed) of the compact group $\mathbb{T}^{G}$.

Clearly, $\sigma(\Gamma G, G) \subset \sigma\left(G^{\wedge}, \Gamma G^{\wedge}\right)$. The latter is the Bohr topology of the dual group $G^{\wedge}$.

The following known fact is a group analogue of the Alaoglu-Bourbaki theorem.

Proposition 6. Let $G$ be a topological Abelian group and let $U$ be a neighborhood of the neutral element of $G$. Then $U^{\triangleright}$ is a compact subset of $\Gamma_{\sigma} G$. Moreover, $U^{\triangleright}$ is an equicontinuous set of characters and therefore it is also compact in $G^{\wedge}$.

Proof. It can be easily seen that $U^{\triangleright}$ is closed in the compact space $\mathbb{T}^{G}$. The rest of the proof is standard.

In the next proposition we collect several important facts.

Proposition 7. Let $G$ be a topological Abelian group.

(a) $\Gamma\left(\Gamma_{\sigma} G\right)=G^{\natural}$.

(b) If $H \subset \Gamma G$ is a subgroup which separates points of $G$, then $H$ is dense in $\Gamma_{\sigma} G$. The converse also holds provided that $G$ is dually separated.

(c) If $S \subset G$ is a subgroup such that $S^{\perp}=\{1\}$, then $S$ is dense in $G^{+}$.

Proof. (a) This is known [9, Corollary 3.8; 11, 27, Theorem 3.1].

(b) Assume otherwise that $H_{1}:=\bar{H} \neq \Gamma G$. Taking into account that $\Gamma_{\sigma} G$ is a totally bounded Abelian group, and that $\Gamma\left(\Gamma_{\sigma} G\right)=G^{\natural}$ (by $(a)$ ), there would exist $g \in G$ distinct from the neutral element of $G$ such that $g^{\natural}{ }_{\mid H_{1}}=1$. In particular, 
$\varphi(g)=g^{\natural}(\varphi)=1$ for all $\varphi \in H$, which contradicts the assumption that $H$ separates points of $G$.

(c) Suppose by contradiction that $S_{1}:=\bar{S} \neq G$. Taking into account that $G^{+}$ is a totally bounded Abelian group, and that $\Gamma G^{+}=\Gamma G$ (this is evident), there would exist $\varphi \in \Gamma G$ such that $\varphi \neq 1$ and $\varphi_{\mid S_{1}}=1$. In particular, $\varphi(g)=1$ for all $g \in S$, that is, $\varphi \in S^{\perp}$. This contradicts the assumption that $S^{\perp}=\{1\}$.

The following easy observation will be useful in the sequel.

Proposition 8. Let $G$ be a dually separated group. Then the natural mapping $\alpha:=\alpha_{G}$ is a topological isomorphism between $G^{+}$and $\left(G^{\natural}, \sigma\left(G^{\natural}, \Gamma_{\sigma} G\right)\right)$.

Proof. (i) $\alpha$ is $1-1$ due to the fact that $G$ is a DS-group.

(ii) $\alpha$ is continuous. If $\left(x_{\beta}\right)$ is a $\sigma(G, \Gamma G)$-convergent net in $G$, say $x_{\beta} \longrightarrow x$, then for every $\varphi \in \Gamma G, \alpha\left(x_{\beta}\right)(\varphi)=\varphi\left(x_{\beta}\right) \longrightarrow \varphi(x)=\alpha(x)(\varphi)$. This implies that $\alpha\left(x_{\beta}\right) \longrightarrow \alpha(x)$.

(iii) $\alpha^{-1}: \alpha\left(G^{+}\right) \longrightarrow G^{+}$is also continuous. Let $\alpha\left(x_{\beta}\right)$ be a net convergent to $\alpha(x)$ in $\alpha\left(G^{+}\right) \subset C_{\mathrm{p}}\left(\Gamma_{\sigma} G, \mathbb{T}\right)$. For each $\varphi \in \Gamma G, \alpha\left(x_{\beta}\right)(\varphi) \longrightarrow \alpha(x)(\varphi)$; therefore $\varphi\left(x_{\beta}\right) \longrightarrow \varphi(x), \forall \varphi \in \Gamma G$. Thus $x_{\beta} \longrightarrow x$ in $G^{+}$.

\section{Extensions of the Eberlein-Šmulyan theorem to Abelian topological groups}

Two classes of topological groups angelic with respect to their Bohr topology are provided by Theorems 11 and 13. They are distinct, as can be inferred from Examples 26 and 27 in Section 7. The following propositions are crucial for obtaining them.

Proposition 9. Let $X$ be a topological Abelian group such that

$$
X=\overline{\bigcup_{n=1}^{\infty} K_{n},}
$$

where each $K_{n}$ is a relatively countably compact subset. Then $\Gamma_{\sigma} X$ is strictly angelic.

Proof. Observe that, by Proposition $5, C_{\mathrm{p}}(X, \mathbb{T})$ is strictly angelic. Since $\Gamma_{\sigma} X$ is a topological subspace of $C_{\mathrm{p}}(X, \mathbb{T})$, by Lemma $3(\mathrm{~b})$, it is strictly angelic.

Proposition 9 is not true in general (for example, from Proposition 12, one can obtain that $\Gamma_{\sigma} X$ is not angelic for a discrete group $X$ with $\left.\operatorname{card}(X) \geqslant \mathfrak{c}\right)$. A dual statement can be formulated as follows.

Proposition 10. Let $G$ be a dually separated group such that

$$
\Gamma_{\sigma} G=\overline{\bigcup_{n=1}^{\infty} K_{n}},
$$

where each $K_{n}$ is a relatively countably compact subset of $\Gamma_{\sigma} G$. Then $G^{+}$is strictly angelic. 
Proof. By Proposition $7(\mathrm{a}), \Gamma\left(\Gamma_{\sigma} G\right)=G^{\natural}$. Hence, applying Proposition 9 to $X:=\Gamma_{\sigma} G$, we get $\left(G^{\natural}, \sigma\left(G^{\natural}, \Gamma_{\sigma} G\right)\right)$ is strictly angelic. The fact that $G^{+}$is strictly angelic follows now from Proposition 8 and Lemma 3(a).

The next statement can be thought of as the counterpart of the Eberlein-Šmulyan theorem for Abelian topological groups.

TheOREm 11. Let $G$ be a metrizable dually separated group. Then $G^{+}$is strictly angelic.

Proof. Let $\left\{U_{n}: n \in \mathbb{N}\right\}$ be a countable neighborhood basis of the neutral element. Clearly $\bigcup_{n \in \mathbb{N}} U_{n}^{\triangleright}=\Gamma G$. Since the sets $U_{n}^{\triangleright}$ are compact in the space $\Gamma_{\sigma} G$ (Proposition 6), $\Gamma_{\sigma} G$ is sigma-compact and Proposition 10 applies.

Under the continuum hypothesis, Theorem 11 is the best possible in the class of Hausdorff LCA groups, as we prove next.

Proposition 12. For a Hausdorff locally compact Abelian group $G$, consider the following statements.

(i) $G^{+}$is angelic.

(ii) $G^{+}$is an $\check{S}$-space.

(iii) $G$ is metrizable.

(iv) $G^{+}$is strictly angelic.

Then (iii) $\Longrightarrow$ (iv) $\Longrightarrow$ (i) $\Longrightarrow$ (ii). Under the continuum hypothesis, (ii) $\Longrightarrow$ (iii).

Proof. (iii) $\Longrightarrow$ (iv) by Theorem 11, while (iv) $\Longrightarrow$ (i) is evident.

(i) $\Longrightarrow$ (ii) by Proposition 2 .

The proof of the second assertion, (ii) $\Longrightarrow$ (iii), is carried out as follows.

Step 1: Suppose that $G$ is compact and therefore its Bohr topology coincides with the original. If $G$ were non-metrizable, its topological weight $\kappa$ would be strictly greater than $\aleph_{0}$. This and the continuum hypothesis imply that $\kappa \geqslant \mathfrak{c}$. Then $G$ would contain a homeomorphic copy of the Cantor cube $\{-1,1\}^{\kappa}$ (see $[\mathbf{2 9}]$ ), which is compact, but not sequentially compact (the proof of this fact follows the same pattern as that of Example 28). This contradicts (ii).

Step 2: Let $G$ be a Hausdorff locally compact Abelian group. There then exists a closed subgroup $H$ of $G$ containing an open compact subgroup of $G$, say $K$, such that $G \cong \mathbb{R}^{n} \times H$, for some $n \in \mathbb{N}$ (see for example [12, Theorem 3.3.10]). Since $G$ is an $\breve{\mathrm{S}}$-space, $K$ is also an $\breve{\mathrm{S}}$-space. Due to compactness, $K=K^{+}$, and by the first step $K$ is metrizable. As $K$ is open in $H$, a countable basis of the zero element in $K$ is also a basis in $H$. Thus $H$ is metrizable and therefore $G$ is metrizable.

TheOREm 13. Let $G$ be a topological Abelian group which contains a neighborhood $U$ of the neutral element such that $U^{\triangleright}$ separates the points of $G$. Then $G^{+}$is strictly angelic. 
Proof. Let $U$ be as in the claim. By Proposition 7(b) and Proposition 6, the group $H$ generated by $U^{\triangleright}$ is dense and sigma-compact in $\Gamma_{\sigma} G$. It then follows from Proposition 10 that $G^{+}$is strictly angelic.

In the framework of locally quasi-convex groups it is possible to obtain a unified version of Theorems 11 and 13, which we give next.

TheOREM 14. Let $G$ be a locally quasi-convex topological Abelian group which admits a coarser metrizable topology $\mathcal{T}_{1}$. Then $G^{+}$is strictly angelic.

Proof. Let $\left\{U_{n}: n \in \mathbb{N}\right\}$ be a countable $\mathcal{T}_{1}$-neighborhood basis of the neutral element 0 . Since $\mathcal{T}_{1}$ is coarser than the original topology of $G$, for each natural $n$ there exists a quasi-convex neighborhood $V_{n}$ of the neutral element of $G$ such that $V_{n} \subset U_{n}$. Put $H_{0}:=\bigcup_{n \in \mathbb{N}} V_{n}^{\triangleright}$, and let us prove that $H_{0}$ separates the points of $G$. For any $g \in G$ with $g \neq 0$, there exists a natural number $n$ such that $g \notin U_{n}$, and consequently $g \notin V_{n}$. $V_{n}$ being quasi-convex, there exists $\varphi \in V_{n}^{\triangleright} \subset H_{0}$ with $\operatorname{Re}(\varphi(g))<0$. Hence $\varphi(g) \neq 1$.

Denote by $H$ the group generated by $H_{0}$. Clearly $H$ is sigma-compact in $\Gamma_{\sigma} G$, and by Proposition 7(b) it is also dense. Now Proposition 10 can be applied to obtain $G^{+}$is strictly angelic.

Note that Theorem 14 for the case when $G$ is a Hausdorff locally convex space improves the Dieudonne-Schwartz version of the Šmulyan theorem, as quoted in [22, Theorem 9.8.2, p. 191].

\section{Groups which are g-spaces with respect to their Bohr topologies}

So far, we have studied some conditions on a topological group $G$ which ensure that $G^{+}$is angelic or strictly angelic. We focus now on the problem of when $G^{+}$ is a $g$-space, in other words, $G^{+}$is only required to satisfy the first condition of angelicity. A bigger class of topological groups is obtained, which in particular contains the complete locally convex spaces. This is the right direction to take to generalize the Eberlein-Šmulyan theorem to the class of locally convex spaces, as was done by Grothendieck in [18, corollary to Theorem 2, p. 209], where some assumption on completeness is needed. We intend to go beyond this, to the class of locally quasi-convex groups, and therefore completeness must appear somewhere.

Let us consider the following property, which a topological Abelian group $G$ may or may not have.

\section{A character $\psi: \Gamma G \longrightarrow \mathbb{T}$, whose restriction to every equicontinuous}

subset $H$ of $\Gamma G$ is $\sigma(\Gamma G, G)_{\left.\right|_{H}}$-continuous, is $\sigma(\Gamma G, G)$-continuous.

If $G$ is in particular a locally convex space, $G$ is complete if and only if it satisfies $(*)$. This follows directly from Grothendieck's completeness criterion. In previous work [7] (see Theorem 4.1), we obtained $(*)$ is a sufficient condition of completeness in the class of Hausdorff locally quasi-convex topological Abelian groups. In the same paper it was proved that there exists a complete metrizable separable locally quasi-convex group which does not satisfy $(*)$. 
On the basis of these considerations, we introduce a new notion. A topological Abelian group $G$ is demi-complete if it satisfies the following condition.

$A$ character $\psi: \Gamma G \longrightarrow \mathbb{T}$, whose restriction to every angelic-producing subspace $H$ of $\Gamma_{\sigma} G$ is $\sigma(\Gamma G, G)_{\left.\right|_{H}}$-continuous, is $\sigma(\Gamma G, G)$-continuous.

Observe that any metrizable group $G$ is demi-complete (the whole $\Gamma_{\sigma} G$ is then angelic-producing, since it is sigma-compact and Proposition 5 applies). Also, the additive group $G$ of any complete locally convex space is demi-complete (by Grothendieck's criterion, since every $\sigma(\Gamma G, G)$-closed equicontinuous subset $H$ of $\Gamma G$ is by Proposition 6 a compact subspace of $\Gamma_{\sigma} G$, and Proposition 5 applies).

THEOREM 15. Let $G$ be a demi-complete dually separated group, and let $K \subset$ $G$. If $K$ is relatively countably compact in $G^{+}$, then it is also relatively compact in $G^{+}$. In other words, $G^{+}$is a $g$-space.

Proof. Put $K^{\natural}:=\left\{g^{\natural}: g \in K\right\}$ and let $F$ be the closure of $K^{\natural}$ in $\mathbb{T}^{\Gamma G}$. Since $F$ is compact in $\mathbb{T}^{\Gamma G}$, it is sufficient to show that $F \subset G^{\natural}$ (then, by Proposition 8 , the set $\alpha_{G}^{-1}(F)$ will be a compact subset of $G^{+}$containing $\left.K\right)$. Fix an arbitrary $\psi \in F$. Clearly $\psi$ is a character on $\Gamma G$. Therefore, by Proposition $7\left(\right.$ a), we will have $\psi \in G^{\natural}$ if we can show that $\psi$ is continuous on $\Gamma_{\sigma} G$. To this end, take any $H \subset \Gamma G$ which is angelic-producing as a subspace of $\Gamma_{\sigma} G$. Since $G$ is demi-complete, the continuity of $\psi$ on $\Gamma_{\sigma} G$ will follow from the continuity of $\psi_{\mid H}$ with respect to $\sigma(\Gamma G, G)_{\mid H}$.

Set $K^{\natural}{ }_{\mid H}:=\left\{g^{\natural}{ }_{\mid H}: g \in K\right\}$. Since $K$ is relatively countably compact in $G^{+}$, we have $K^{\natural}{ }_{\mid H}$ is relatively countably compact in $C_{\mathrm{p}}(H, \mathbb{T})$. Taking into account the fact that $H$ is angelic-producing, the space $C_{\mathrm{p}}(H, \mathbb{T})$ is angelic and therefore $K^{\natural}{ }_{\mid H}$ is relatively compact in $C_{\mathrm{p}}(H, \mathbb{T})$. It follows that the closure of $K^{\natural}{ }_{\mid H}$ in $\mathbb{T}^{H}$ is contained in $C_{\mathrm{p}}(H, \mathbb{T})$. Since $\psi$ belongs to the closure of $K^{\natural}$ in $\mathbb{T}^{\Gamma G}$, we have $\psi_{\mid H}$ belongs to the closure of $K^{\natural}{ }_{\mid H}$ in $\mathbb{T}^{H}$. Consequently, $\psi_{\mid H} \in C_{\mathrm{p}}(H, \mathbb{T})$, and this means that $\psi_{\mid H}$ is continuous with respect to $\sigma(\Gamma G, G)_{\mid H}$.

Next we deal with locally convex vector groups. A locally convex vector group is defined as a vector space endowed with a topology which makes addition continuous and which has a local basis for the neutral element formed by convex symmetric subsets. For this class of objects, which is between the classes of locally convex spaces and of locally quasi-convex groups, there is full equivalence between completeness and $(*)[\mathbf{7}$, Corollary 4.4$]$. Therefore a complete locally convex vector group is demi-complete, and by Theorem 15 we have the following corollary.

Corollary 16. If $G$ is a complete locally convex vector group, then $G^{+}$is a g-space.

Notice that Theorem 15 gives a property weaker than angelicity for a big class of topological groups. A better result cannot be expected, even for the additive group of a complete locally convex space. In fact, an uncountable product of real lines is not angelic.

We do not know whether Corollary 16 remains true for any complete locally quasiconvex group. We will show that, even with milder assumptions, the assertion holds for nuclear groups (Proposition 18). The definition as well as a thorough study of 
nuclear groups is presented in [3]. Two outstanding properties of the class of nuclear groups are the following: (i) it contains all the LCA groups and all the nuclear locally convex spaces considered in their group structure, and (ii) it is closed by the operations of taking subgroups, Hausdorff quotients, arbitrary products and countable direct sums. The following assertions will be used in the sequel.

Lemma 17. Let $G$ be a nuclear group. Then the following hold.

(a) Every closed subgroup of $G$ is closed in $G^{+}[\mathbf{3},(8.6)]$.

(b) Every compact subset of $G^{+}$is also compact in $G[4]$.

(c) Every relatively countably compact subset of $G^{+}$is totally bounded in $G[4]$.

If a group $G$ is such that any closed totally bounded subset of $G$ is compact, we shall say that $G$ is von Neumann-complete. Clearly any complete group is von Neumann-complete. The converse holds for a metrizable group, but not in general $[28]$.

The following is a stronger version of Corollary 16.

Proposition 18. If $G$ is a Hausdorff von Neumann-complete nuclear group, then $G^{+}$is a $g$-space. Even more is true: if $K$ is any relatively countably compact subset of $G^{+}$, then $K$ is relatively compact not only in $G^{+}$, but in $G$ too.

Proof. By Lemma $17(\mathrm{c}), K$ is totally bounded in the original topology of $G$. Since $G$ is von Neumann-complete, $K$ is relatively compact in $G$. On the other hand, $G^{+}$is Hausdorff, due to the fact that $G$ is a Hausdorff nuclear group, and therefore a DS-group. Thus we see that $K$ is relatively compact in $G^{+}$.

Notice that Proposition 18 is not true for all nuclear groups. In Example 29, we describe a totally bounded Hausdorff topological Abelian group $\mathbf{M}$ such that $\mathbf{M}^{+}=\mathbf{M}$ is not a $g$-space.

\section{NSS-groups}

In this section we shall study in more detail the class of groups for which Theorem 13 is applicable. A topological group $G$ is said to have no small subgroups (or to be an NSS-group) if there is a neighborhood of the neutral element which contains no nontrivial subgroup of $G$. This is a significant property, being linked with the solution of the fifth problem of Hilbert. After the positive answer to the latter given by Gleason, Montgomery and Zippin for locally euclidean groups, Yamabe proved that in general, a locally compact group which is an NSS-group admits an analytic structure which makes it a Lie group. Reasonably, the property may also be of interest outside of the class of locally compact groups, and it has appeared in our work in a natural way, during the searching of Bohr angelic groups.

In the sequel we shall use the following two assertions, whose proof derives easily from the fact that $\mathbb{T}_{+}:=\{t \in \mathbb{T}: \operatorname{Re}(t) \geqslant 0\}$ does not contain any nontrivial subgroup of $\mathbb{T}$ (thus $\mathbb{T}$ is an NSS-group).

Lemma 19. Let $M$ be a nonempty subset of $G$. Then the following hold.

(a) If $M$ contains a subgroup $H$, then $\varphi(H)=\{1\}$ for all $\varphi \in M^{\triangleright}$.

(b) If $M^{\triangleright}$ contains a subgroup $L$, then $\varphi(M)=\{1\}$ for all $\varphi$ in $L$. 
The equivalence we state next was known to hold for locally compact Abelian Hausdorff groups (LCA groups) [2, Proposition 7.9]. We extend it to a larger class, which contains all the groups whose closed subgroups are also Bohr-closed, and all the nuclear groups thereof (Lemma 17(a)).

Proposition 20. For a topological Abelian group $G$, consider the next two claims.

(a) $G$ contains a compactly generated dense subgroup.

(b) The dual $G^{\wedge}$ has no small subgroups.

Then (a) implies (b). The converse also holds, provided that any dense subgroup of $G^{+}$is also dense in $G$.

Proof. (a) $\Longrightarrow$ (b) Let $K$ be a compact subset of $G$ which spans a dense subgroup $S$. Suppose that $L$ is a subgroup contained in $K^{\triangleright}$. For any $\varphi$ in $L$, by Lemma 19(b), $\varphi(K)=\{1\}$, and hence $\varphi(S)=\{1\}$. By the density of $S, \varphi(G)=\{1\}$ also, which means that $L$ contains only the null character. Thus $K^{\triangleright}$ is a 0 -neighborhood in $G^{\wedge}$, which contains no nontrivial subgroup of $G^{\wedge}$.

For the second statement, now let $K$ be a compact subset of $G$ such that $K^{\triangleright}$ does not contain nontrivial subgroups. Denote by $S$ the subgroup of $G$ generated by $K$. Since $K^{\perp}$ is a subgroup contained in $K^{\triangleright}, K^{\perp}=\{1\}$, and hence $S^{\perp}=\{1\}$. By Proposition $7(\mathrm{c}), S$ is dense in $G^{+}$, and by the assumption made, $S$ is also dense in $G$.

Proposition 21. Let $G$ be a topological Abelian group and let $U$ be a neighborhood of the neutral element of $G$. Then $U^{\triangleright}$ separates the points of $G$ if and only if the quasi-convex hull of $U$ contains no nontrivial subgroups.

Proof. The quasi-convex hull of $U$ always contains the subgroup $\bigcap_{\varphi \in U^{\triangleright}} \varphi^{-1}(1)$. Therefore, under the assumption that $Q(U)$ does not contain nontrivial subgroups, $\bigcap_{\varphi \in U^{\triangleright}} \varphi^{-1}(1)=\{0\}$. In particular, for every $g \neq 0$, there exists $\varphi \in U^{\triangleright}$ such that $\varphi(g) \neq 1$.

Conversely, if $Q(U)$ contains a nontrivial subgroup $H$, then $\varphi(H)=1$ for all $\varphi \in U^{\triangleright}$. Thus $U^{\triangleright}$ does not separate the points of $G$.

The next statement is a dual version of Proposition 20.

TheOREM 22. Let $G$ be a reflexive group such that every closed subgroup of its dual $G^{\wedge}$ is Bohr-closed (in particular, $G$ may be a Čech-complete nuclear group). It is sufficient to assume that every dense subgroup of $\Gamma_{\sigma} G$ is dense in $G^{\wedge}$. The following assertions are equivalent.

(a) $G$ contains a neighborhood $U$ of the neutral element of $G$ such that $U^{\triangleright}$ separates the points of $G$.

(b) $G$ has no small subgroups.

(c) $G^{\wedge}$ contains a dense compactly generated subgroup.

Proof. The equivalence between (a) and (b) can be obtained from Proposition 21, taking into account the fact that a reflexive group is locally quasi-convex. On the other hand, applying Proposition 20 to $G^{\wedge}$, we obtain (c) is equivalent to $(b)$. 


\section{Angelicity and the Schur property}

Recall that a group $G$ is said to respect compactness if every Bohr compact subset of $G$ is compact. Therefore, by Lemma 17(b), any nuclear group respects compactness.

Next we prove that for the groups which are angelic in their Bohr topology, respecting compactness can be expressed in terms of sequences. We need the following notion: a topological Abelian group $G$ has the Schur property if every convergent sequence of $G^{+}$is convergent in $G$.

Proposition 23. Let $G$ be a dually separated topological Abelian group. Consider the following assertions.

(a) $G$ respects compactness.

(b) $G$ has the Schur property.

Then the following hold.

(1) (a) implies (b).

(2) If $G$ has the Schur property and $K$ is a sequentially compact subset of $G^{+}$, then $K$ is sequentially compact in $G$.

(3) If $G$ has the Schur property and $K$ is a compact subset of $G^{+}$, then $K$ is compact in $G$ provided that at least one of the following conditions is satisfied.

(C1) $G^{+}$is angelic.

(C2) $G^{+}$is an $\breve{S}$-space and $G$ is von Neumann-complete.

(C3) $G^{+}$is an $\check{S}$-space and $G$ is locally quasi-convex.

Proof. (1) Take a sequence $\left(x_{n}\right)$ in $G$ which is Bohr-convergent to $x \in G$. The set $S=\left\{x_{n}\right\} \cup\{x\}$ is Bohr-compact, and by (a) is also compact. Let $y \in G$ be a cluster point of $\left(x_{n}\right)$ in $G$. Then $y$ is also a cluster point of $\left(x_{n}\right)$ in $G^{+}$. Since $G^{+}$ is separated, we get $y=x$. This and compactness of $S=\left\{x_{n}\right\} \cup\{x\}$ imply that $x_{n} \longrightarrow x$ in $G$.

(2) In fact, take a sequence $\left(x_{n}\right)$ in $K$. Since $K$ is Bohr sequentially compact, $\left(x_{n}\right)$ has a Bohr-convergent subsequence $\left(x_{p_{n}}\right)$. By the Schur property of $G,\left(x_{p_{n}}\right)$ converges in $G$ too. Therefore $K$ is sequentially compact in the original topology of $G$.

(3) (i) Suppose that (C1) is satisfied. By Lemma 3(d), $G$ is angelic too. From this, Proposition 1 and (2), we get $K$ is compact.

(ii) Suppose that (C2) is satisfied. Then $K$ is Bohr sequentially compact and by (2), it is sequentially compact. This implies that $K$ is totally bounded as a subset of $G[\mathbf{1 4}, 1.2(3)$, p. 7]. The Bohr compactness of $K$ implies that $K$ is a closed subset of $G$. Since $G$ is von Neumann-complete, its closed and totally bounded subset $K$ is compact.

(iii) Suppose that (C3) is satisfied. As in the previous case, we can conclude that $K$ is a totally bounded subset of $G$. Since it is Bohr-compact, it is complete in $\sigma(G, \Gamma G)$. It is also complete in the original topology of $G$, due to the fact that it has a basis at zero of quasi-convex neighborhoods, which are in particular Bohr-closed. Therefore $K$ is a compact subset of $G$. 
Corollary 24. Let $G$ be a dually separated topological Abelian group. If $G^{+}$ is angelic, then the following statements are equivalent.

(a) $G$ respects compactness.

(b) $G$ has the Schur property.

REMARK 25. The implication (b) $\Longrightarrow$ (a) is not valid in general (a way of constructing a corresponding example is indicated in [30, Example 6, p. 267], and a complete proof is given in [13, Example 19.19, p. 116-117]).

\section{Some examples}

EXAMPLE 26 (a compact metrizable Abelian group $G$, such that for every neighborhood $V$ of the neutral element, $V^{\triangleright}$ does not separate the points of $G$ ). The product of countably many copies of $\mathbb{T}$, say $G:=\mathbb{T}^{\mathbb{N}}$, is compact metrizable. However, the polar of any neighborhood of zero $V \subset G$ does not separate points.

In fact, let $V=\bigcap_{i \in F} p_{i}^{-1}\left(U_{i}\right)$, being $U_{i} \in \mathcal{B}_{\mathbb{T}}(1)$ for all $i$ in a finite set $F \subset \mathbb{N}$. Clearly $V$ contains the subgroup $P:=\{1\} \times \ldots \times \mathbb{T} \times\{1\} \times \ldots$, where the factors $\mathbb{T}$ are located in the $j$-coordinates, for $j \notin F$. If $\varphi \in V^{\triangleright}, \varphi(P)=1$.

EXAMPLE 27 (a noncompact nonmetrizable Abelian group $G$, which contains a neighborhood $U$ of the neutral element such that $U^{\triangleright}$ separates the points of $G$ ). Let $G:=\omega \mathbb{R}$ be the direct sum of countably many copies of $\mathbb{R}$ with the topology induced by the box topology of $\mathbb{R}^{\mathbb{N}}$. The fact that $G$ is nonmetrizable can be easily checked. On the other hand, $G$ meets the requirements of Theorem 22. Firstly, closed subgroups of $G$ are Bohr-closed due to the fact that $G$ is strongly reflexive $[\mathbf{3},(17.1)]$. Secondly, neighborhoods such as $U:=((-1,1) \times(-1,1) \times \ldots) \cap \omega \mathbb{R}$ do not contain nontrivial subgroups.

EXAMPLE 28 ((1) a compact Hausdorff Abelian group which is not sequentially compact, and therefore, not angelic, (2) A sequentially compact Hausdorff Abelian group $S$ which is not compact). (1) Let $G:=\mathbb{T}^{\mathbb{R}}$. Clearly $G$ is compact Hausdorff. Now we want to find a sequence without convergent subsequences. Take a bijection $g: \mathbb{R} \longrightarrow \mathbb{N}^{\mathbb{N}}$, and put $g(r)=\left(j_{1}^{r}, j_{2}^{r}, \ldots, j_{n}^{r}, \ldots\right)$. Define $x_{n}=\left(x_{n}(r)\right)_{r \in \mathbb{R}} \in \mathbb{T}^{\mathbb{R}}$ by

$$
x_{n}(r):= \begin{cases}i \in \mathbb{T} & \text { if } n=j_{k}^{r} \text { for some odd } k \\ 1 \in \mathbb{T} & \text { if } n \notin\left\{j_{1}^{r}, j_{3}^{r}, \ldots, j_{2 p+1}^{r}, \ldots\right\} .\end{cases}
$$

The sequence $\left\{x_{1}, x_{2}, \ldots, x_{n}, \ldots\right\} \subset \mathbb{T}^{\mathbb{R}}$ does not have convergent subsequences; in fact, were $\left\{x_{l_{m}}\right\} \subset\left\{x_{n}\right\}$ a convergent subsequence, then $\left\{x_{l_{m}}(r)\right\}$ would also be convergent for every $r \in \mathbb{R}$. Suppose that $s \in \mathbb{R}$ is such that $g(s)=\left(l_{m}\right)$. Then $\left\{x_{l_{m}}(s)\right\}=\{i, 1, i, 1, \ldots\}$ is not convergent in $\mathbb{T}$, which contradicts the previous statement.

(2) Let $G:=\mathbb{T}^{\mathbb{R}}$. For $x \in G$, denote $\operatorname{supp}(x)=\{r \in \mathbb{R}: x(r) \neq 1\}$ and

$$
S:=\left\{x \in G: \operatorname{card}(\operatorname{supp}(x)) \leqslant \aleph_{0}\right\} .
$$

Then $S$ is a dense subgroup of $G$. It follows that $S$ with the topology induced from $G$ is sequentially compact, since the range of any sequence can be embedded in a countable product of copies of $\mathbb{T}$, which is obviously metrizable. (The space $S$ 
is frequently called a $\Sigma$-product. Notice that the Stone-Čech compactification $\beta S$ coincides with $\mathbb{T}^{\mathbb{R}}$; see $[\mathbf{1 5}, \mathbf{2 3}]$ ).

EXAMPLE 29 (a totally bounded Hausdorff Abelian group $\mathbf{M}$ which is not countably compact but contains a dense sequentially compact subgroup; therefore, $\mathbf{M}$ is not a $g$-space). Let $K$ be the discrete multiplicative group $\{-1,1\}$ and let $G:=K^{\mathbb{R}}$. Fix a sequence $I_{n}, n=1,2, \ldots$ of disjoint uncountable subsets of $\mathbb{R}$ such that $\mathbb{R}=\bigcup_{n=1}^{\infty} I_{n}$. Let

$$
\mathbf{M}:=\left\{x \in G: \exists n \in \mathbb{N}, \operatorname{card}\left(\operatorname{supp}(x) \cap \bigcup_{k=n}^{\infty} I_{k}\right) \leqslant \aleph_{0}\right\} .
$$

As in the previous example, denote by $S$ the $\Sigma$-product of $\mathbb{R}$-copies of $K$. Evidently $S \subset \mathbf{M}$, and $\mathbf{M}$ is a totally bounded subgroup of the Cantor group $G$. The closure of $S$ in $\mathbf{M}$ is precisely $\mathbf{M}$ (take into account the fact that $S$ is dense in $G$ ). Therefore $\mathbf{M}$ contains a dense sequentially compact subgroup.

Let us prove that $\mathbf{M}$ is not countably compact. For each natural $n$, put $J_{n}=$ $\bigcup_{k=1}^{n} I_{k}$ and define a mapping $f_{n}: \mathbb{R} \longrightarrow K$ as follows: $f_{n}(r)=-1, \forall r \in J_{n}$, and $f_{n}(r)=1$ otherwise. Clearly $f_{n} \in \mathbf{M}$ for all $n \in \mathbb{N}$ and $f_{n} \rightarrow-\mathbf{1}$ pointwise in $G$, where $\mathbf{- 1}$ denotes the function with constant value -1 . Since $\mathbf{- 1} \notin \mathbf{M}$, the sequence $\left\{f_{n}\right\}$ does not have any cluster point in $\mathbf{M}$.

REmark 30. The idea of Example 29 is taken from [14, Example 1.2(9), p. 9]; we have corrected a gap in the reference.

Acknowledgements. The authors are grateful to the referee for his thorough reading of the paper, and his keen suggestions for improving it. We also thank Professor D. Dikranjan for pointing out to us that we could drop 'compactly generated' from Proposition 12, as we had in a previous version of the paper.

\section{References}

1. A. V. Arhangel'Skit, 'On a theorem of Grothendieck in $C_{p}$-theory', Topology Appl. 80 (1997) $21-41$.

2. D. L. Armacost, The structure of locally compact Abelian groups (Marcel Dekker, New York, 1981).

3. W. BANASzczyK, Additive subgroups of topological vector spaces, Lecture Notes in Mathematics 1466 (Springer, 1991).

4. W. BAnAszczyK and E. Martín-Peinador, 'The Glicksberg theorem on weakly compact sets for nuclear groups', Annals of the New York Academy of Sciences 788 (New York Academy of Sciences, New York, 1996) 34-39.

5. T. K. Boenme and M. Rosenfeld, 'An example of two compact Hausdorff Frechet spaces whose product is not Frechet', J. London Math. Soc. (2) 8 (1974) 339-344.

6. M. Bruguera, 'Grupos topológicos y grupos de convergencia: estudio de la dualidad de Pontryagin', Doctoral Dissertation, Universidad de Barcelona, 1999.

7. M. Bruguera, M. J. Chasco, E. Martín-Peinador and V. Tarieladze, 'Completeness properties of locally quasi-convex groups', Topology Appl. 111 (2001) 81-93.

8. B. Cascales and J. ORinuela, 'On compactness in locally convex spaces', Math. Z. 195 (1987) 365-381.

9. M. J. Chasco, E. Martín-Peinador and V. Tarieladze, 'On Mackey topology for groups', Studia Math. 132 (1999) 257-284.

10. W. W. Comfort and K. A. Ross, 'Topologies induced by groups of characters', Fund. Math. (1964) 191-283.

11. W. W. Comfort, S. Hernández and F. J. Trigos-Arrieta, 'Relating a locally compact Abelian group to its Bohr compactification', Adv. Math. 120 (1996) 322-344. 
12. D. N. Dikranjan, I. R. Prodanov and L. N. Stoyanov, Topological groups: characters, dualities and minimal group topologies, Monographs and Textbooks in Pure and Applied Mathematics 130 (Marcel Dekker, New York, 1990).

13. X. Domínguez, 'Grupos Abelianos topológicos y sumabilidad', Doctoral Dissertation, Universidad Complutense de Madrid, 2001.

14. K. Floret, Weakly compact sets, Lecture Notes in Mathematics 801 (Springer, 1980).

15. I. Glicksberg, 'Stone-Cech compactifications of products', Trans. Amer. Math. Soc. 90 (1959) 369-382.

16. W. Govaerts, 'A productive class of angelic spaces', J. London Math. Soc. (2) 22 (1980) $355-364$.

17. A. Grothendieck, 'Critères de compacité dans les espaces fonctionells généraux', Amer. J. Math. 74 (1952) 168-186.

18. A. Grothendieck, Topological vector spaces (Gordon \& Breach, New York, 1973).

19. R. Haydon, 'Compactness in $C_{s}(T)$ and applications', Publ. Dép. Math. 9 (1972) 105-113.

20. S. Hernández, J. Galindo and S. Macario, 'A characterization of the Schur property by means of the Bohr topology', Topology Appl. 97 (1999) 99-108.

21. M. Ismail and P. Nyikos, 'On spaces in which countably compact sets are closed', Topology Appl. 11 (1980) 281-292.

22. H. JARCHOW, Locally convex spaces (Teubner, Stuttgart, 1981).

23. J. M. Kister, 'Uniform continuity and compactness in topological groups', Proc. Amer. Math. Soc. 13 (1962) 37-40.

24. E. Martín-Peinador and V. Tarieladze, 'Properties of Dunford-Pettis type in topological groups', Proc. Amer. Math. Soc. 132 (2004) 1827-1837.

25. J. Orihuela, 'Pointwise compactness in spaces of continuous functions', Proc. London Math. Soc. (3) 36 (1987) 143-152.

26. J. D. PRYCE, 'A device of R. J. Whitley's applied to pointwise compactness in spaces of continuous functions', Proc. London Math. Soc. (3) 23 (1971) 532-546.

27. S. U. RACZkowski and F. J. Trigos-Arrieta, 'Duality of totally bounded Abelian groups', Bol. Soc. Mat. Mexicana 7 (2001) 1-12.

28. M. RAJAgopalan and R. F. WheEler, 'Sequential compactness of $X$ implies a completeness property for $C(X)$ ', Canad. J. Math. XXVIII (1976) 207-210.

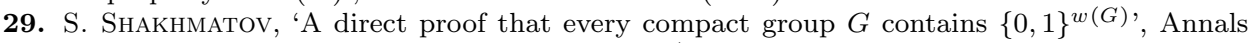
of the New York Academy of Sciences 728 (New York Academy of Sciences, New York, 1994) 276-283.

30. A. Wilansky, Modern methods in topological vector spaces (McGraw-Hill, 1978).

Montserrat Bruguera

Departament de Matemàtica Aplicada $I$

Universitat Politècnica

de Catalunya

08028 Barcelona

Spain

m.montserrat.bruguera@upc.es

Vaja Tarieladze

Muskhelishvili Institute of Computational Mathematics

Georgian Academy of Sciences

8 Akuri Str.

Tbilisi 380093

Georgia

tar@gw.acnet.ge

\author{
Elena Martín-Peinador \\ Departamento de Geometría y Topología \\ Universidad Complutense de Madrid \\ 28040 Madrid \\ Spain \\ peinador@mat.ucm.es
}

